\title{
Editorial
}

Journal of Innate

Immunity

\section{Autophagy and Innate Immunity}

\author{
Véronique Witko-Sarsat ${ }^{a}$ Patrice Codogno ${ }^{b}$ \\ aINSERM U1016, Cochin Institute, CNRS UMR8104, Université Paris Descartes, Université Paris Diderot, Sorbonne

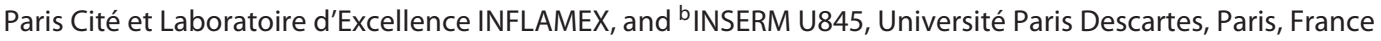

Different and timely aspects of the role of autophagy and autophagy proteins in innate immunology are reviewed by leading authors in the field in this issue of the Journal of Innate Immunity. Indeed, autophagy has recently turned out to play a critical role in host defense responses by promoting the elimination of pathogens and the induction of acquired immunity.

Macroautophagy (hereafter referred to as autophagy) is a form of autophagy that starts with the biogenesis of a double membrane-bound autophagosome that sequesters a fraction of the cytoplasm in a bulk or in a selective manner to deliver cargo to the lysosome [1]. Because autophagy is the only known mechanism to remove cytoplasmic contents that are larger than can be captured by the proteasome, it has also been proposed as a likely component of the cell's arsenal against infectious organisms. Indeed, protein degradation is critically involved in the control of innate immune responses.

Autophagosome formation is under the control of Atg (autophagy-related) genes that are conserved during eukaryotic evolution. Recent advances in the study of Atgs provide strong evidence that they influence both the host defense response and the regulation of inflammation. Consistently, it has been proposed that the compromised function of autophagy regulators [ATG16L1, IRGM (im- munity-related GTPase M) and NOD2 (nucleotide-binding oligomerization domain 2)] results in the development of immune-related diseases, such as Crohn's disease, a chronic inflammatory bowel disease [2].

Autophagy represents an ancient immune defense strategy present in flies. Importantly, as flies lack an adaptive immune system, the function of autophagy in cellintrinsic innate immunity can be studied in isolation without the added complexity of adaptive immunity. In addition to the autophagic pathway, innate immune pathways are conserved between flies and mammals. The use of flies to interrogate immune defense pathways has significantly enhanced our understanding of antimicrobial autophagy due in large part to the sophisticated genetic tools available in flies [3]. Beyond this conservation, it should be emphasized that signaling pathways that control innate immunity also regulate autophagy. In recent years, the autophagy membrane trafficking itself has been analyzed in terms of targeting, docking and fusion membrane events. Deciphering the signaling associated with membrane trafficking of infectious microorganisms in relation to autophagy during infection will help to better understand the tuning of the immune response [4]. The notion of autophagy as a purely degradative pathway was recently challenged by the emergence of studies that have

\section{KARGER}

E-Mail karger@karger.com

www.karger.com/jin (c) 2013 S. Karger AG, Basel

$1662-811 \mathrm{X} / 13 / 0055-0425 \$ 38.00 / 0$
Dr. Veronique Witko-Sarsat

Department of Immunology-Hematology, Cochin Institute, INSERM U1016

27, rue du Faubourg Saint-Jacques

FR-75014 Paris (France)

E-Mail veronique.witko@inserm.fr 
assigned a non-degradative function to autophagy, manifested as unconventional protein secretion exemplified by the interleukin- $1 \beta$ secretion and inflammasome activation [5].

Autophagy is likely one of the most ancient cell-autonomous defense mechanisms to confront microbial invasion. There are complex interactions between invasive organisms and autophagy, suggesting that antimicrobial autophagy has exerted strong evolutionary pressures on pathogens. Selective forms of autophagy can fight invad- ers and can serve as an antiviral defense. However, some pathogens have evoked mechanisms to use or escape autophagy sequestration, and autophagy can be used for viral replication. Moreover, it is clear now that autophagy proteins can play a role during viral infection beyond their role in autophagy [6]. Whether modulation of autophagy may represent a novel therapeutic strategy for treating diseases caused by clinically important pathogens will be discussed.

\section{References}

1 Klionsky DJ, Codogno P: The mechanism and physiological function of macroautophagy. J Innate Immun 2013;5:427-433.

$>2$ Nguyen HTT, Lapaquette P, Bringer M-A, Darfeuille-Michaud A: Autophagy and Crohn's disease. J Innate Immun 2013;5:434443.
Moy RH, Cherry S: Antimicrobial autophagy: a conserved innate immune response in Drosophila. J Innate Immun 2013;5:444-455.

4 Faure M, Lafont F: Pathogen-induced autophagy signaling in innate immunity. J Innate Immun 2013;5:456-470.
Jiang S, Dupont N, Castillo EF, Deretic V: Secretory versus degradative autophagy: unconventional secretion of inflammatory mediators. J Innate Immun 2013;5:471-479.

6 Dong X, Levine B: Autophagy and viruses: adversaries or allies? J Innate Immun 2013;5: 480-493. 